\title{
Systematic analysis about the polarization in thermal radiations of magnetars
}

\author{
Akihiro Yatabe and Shoichi Yamada \\ Advanced Research Institute for Science and Engineering, Waseda University, 3-4-1, Okubo, \\ Shinjuku, Tokyo 169-8555, Japan \\ email: yatabe@heap.phys.waseda.ac.jp
}

\begin{abstract}
We calculated the polarization in soft X-ray emitted from magnetars, which are expected to be observed by the next-generation X-ray satellites. We consider possible conversions of photon's polarization modes in the atmosphere and it cannot be ignored when the magnetic field is relatively weak $B \lesssim 10^{13} \mathrm{G}$.
\end{abstract}

Keywords. magnetic fields, polarization, stars:neutron.

\section{Introduction}

X-ray polarimetry will be realized in the near future. In fact, the Imaging X-ray Polarimetry Explorer (IXPE) (Weisskopf et al. 2013) is planned to be launched in 2020. One of the objectives in some proposed observations in the soft X-ray band, $\sim$ a few $\mathrm{keV}$, is the thermal radiations of neutron stars. If the polarization of these thermal radiations is observed, it will provide us with an important clue to the physical properties of neutron stars near the surface as well as the possible configurations of their magnetic fields. Another target of the polarimetry is the validation of strong-field quantum electrodynamics (QED), a quantum theory for electrons and photons in the electromagnetic fields with strengths of $\gtrsim 4.4 \times 10^{13} \mathrm{G}$ in the case of magnetic fields. Recently, a hint of the vacuum polarization effect is obtained in the optical observation of polarizations in the thermal emissions from an XDINS (Mignani et al. 2017).

\section{Method}

X-ray photons have two polarized normal modes, i.e., $E$-mode and $O$-mode and the $E$-mode photons are dominant in the emergent photons. The $E$-mode $(O$-mode) photons in the plasma-dominant regime have the same helicity as the $O$-mode ( $E$-mode) photons in the vacuum-dominant regime. As a result of this property, when a photon propagates from the inner atmosphere of neutron star, where the plasma effect is dominant, through the outer part to the magnetosphere, where the vacuum effect is dominant, the so-called mode conversion may occur from the $E$-mode photon to the $O$-mode and vice versa. The mode conversion occurs adiabatically when $E \gg E_{\mathrm{ad}} \sim 2 \mathrm{keV}$ is satisfied, where $E$ is the photon energy (Lai \& Ho (2003)). The mode conversion may modify the polarization produced in the surface radiation. When the magnetic field satisfies $B<B_{l} \sim 5 \times$ $10^{13} \mathrm{G}$, we assume that the $O$-mode, into which the originally dominant $E$-mode is converted, becomes predominant as long as the mode conversion occurs adiabatically. On the other hand, if $B>B_{l}$ is satisfied, we assume that the mode conversion does not occur and that the $E$-mode is dominant. The polarization is further modified in the magnetosphere (Heyl \& Shaviv (2002)). We assume that the polarization is essentially fixed at the point corresponding to the so-called polarization-limiting radius (Taverna 

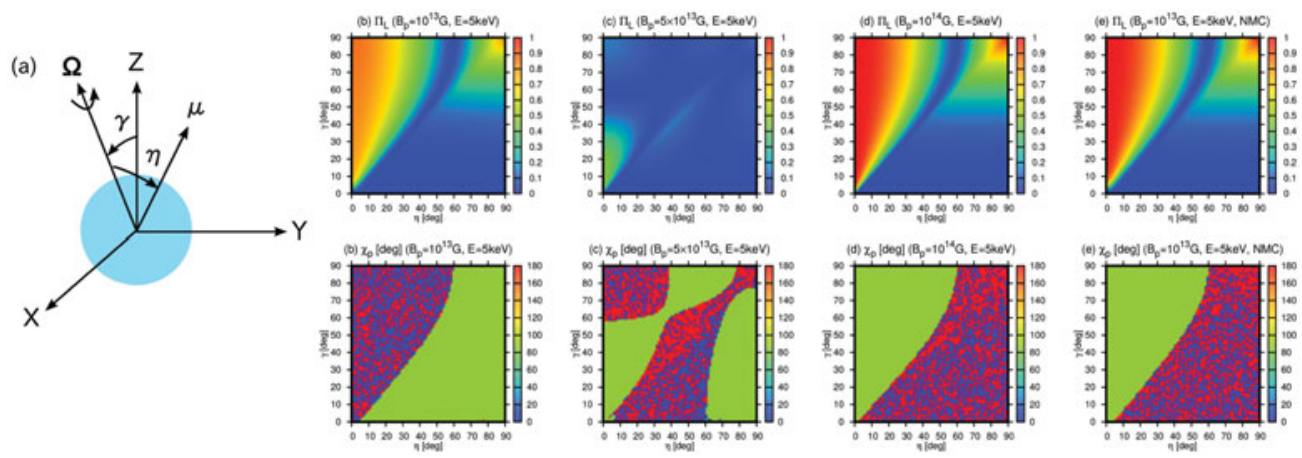

Figure 1. Panel (a) shows the configuration of a neutron star. Panels (b)-(e) show the phase-averaged polarization fraction (upper panels) and angles (lower panels).

et al. (2015)). We consider only dipole magnetic fields and we introduce coordinates as shown in Fig. 1 (a). In this frame, an observer is assumed to be sitting at an infinite distance on the positive $Z$-axis. To derive the linear polarization fraction and angle, we utilize the Stokes parameters, $I, Q$ and $U$, where $I$ is the intensity and $Q$ and $U$ show the linear polarization. The polarization fraction and angle are derived from the Stokes parameters as $\Pi_{L}=\sqrt{Q^{2}+U^{2}} / I$ and $\chi_{p}=\arctan (U / Q) / 2$, respectively.

\section{Results and Discussions}

We show in Fig. 1 (b)-(e) the four representative patterns of the phase-averaged polarization fraction and angle in the $\eta-\gamma$ plane: (b) $B_{p}=10^{13} \mathrm{G}, E=5 \mathrm{keV}$, (c) $B_{p}=5 \times 10^{13} \mathrm{G}, E=5 \mathrm{keV}$, (d) $B_{p}=10^{14} \mathrm{G}, E=5 \mathrm{keV}$ and $(\mathrm{e}) B_{p}=10^{13} \mathrm{G}, E=5 \mathrm{keV}$ with no mode conversion, where $B_{p}$ is the strength of the dipole magnetic field. Note that the phase-averaged quantities are calculated as the integral of the Stokes parameters over the entire rotational phase. It is apparent that case (d) with the highest magneticfield strength, $B_{p}=10^{14} \mathrm{G}$, is quite similar to case (e). This is the case not only for the polarization fraction but also for the polarization angle and is simply because the mode conversion does not occur in case (d). For the lower magnetic fields, $B_{p}=10^{13}, 5 \times 10^{13} \mathrm{G}$, assumed in cases (b) and (c), the mode conversion is important. In case (b), photons are mostly in the $O$-mode at $E=5 \mathrm{keV}$. As a consequence, the phase-averaged polarization angles are changed by $90^{\circ}$ from case (e). In case (c) with $B_{p}=5 \times 10^{13} \mathrm{G}$, on the other hand, the observer will see both regions with full and no conversions simultaneously. The numbers of $E$-mode and $O$-mode photons are nearly equal to each other in this case and the phase-averaged polarization fractions are severely reduced. The phase-averaged polarization angles have different features in this case. In some parameter regions the polarization angle is seen to rotate by $90^{\circ}$ because of the mode conversion.

\section{References}

Weisskopf, M. C., Baldini, L., Bellazzini, R., et al. 2013, Proc. of SPIE, 8859, 885908

Mignani, R. P., Testa, V., González Caniulef, D., et al. 2017, MNRAS, 465, 492

Lai, D. \& Ho, W. C. G. 2003, ApJ, 588, 962

Heyl, J. S. \& Shaviv, N. J. 2002, Phys. Rev. D, 66, 023002

Taverna, R., Turolla, R., Gonzalez Caniulef, D., et al. 2015, MNRAS, 454, 3254 